\title{
Overexpression of KIF2A is Suppressed by miR-206 and Associated with Poor Prognosis in Ovarian Cancer
}

\author{
Nan Sheng Yun-Zhao Xu Qing-Hua Xi Hai-Yan Jiang \\ Chen-Yi Wang Yu Zhang Qing Ye \\ Department of Obstetrics and Gynecology, Affiliated Hospital of Nantong University, Nantong, China
}

\section{Key Words}

KIF2A • Ovarian Cancer • miR-206 • Proliferation • Migration • Invasion • Apoptosis

\begin{abstract}
Background/Aims: This study aimed to investigate the expression and prognostic value of kinesin family member 2A (KIF2A) and the suppression effects of microRNA-206 (miR206) on KIF2A in ovarian cancer. Methods: Ovarian cancer tissues from patients and ovarian cancer cell lines (A2780 and SKOV3) were used in this study. miR-206 mimics and control were transiently transfected into cells. RT-qPCR was performed to detect KIF2A mRNA and miR-206 expression levels, Western blot was performed to detect KIF2A protein levels, DualLuciferase Reporter Assay was used to examine the inhibition effects of miR-206 on KIF2A mRNA, immunohistochemical staining was used to examine the expression of KIF2A in tissue sections. CCK-8, transwell and Annexin-V-FITC/Propidium lodide staining with flow cytometry were used to detect the cell proliferation, migration/invasion, and apoptosis respectively. Results: Our study explored the expression profiles of KIF2A and miR-206 in the patients with ovarian cancer. We found that overexpression of KIF2A was associated with a poor prognosis in ovarian cancer. We also found that KIF2A mRNA contains two target sites for miR-206 binding and confirmed that miR-206 directly suppresses KIF2A; inhibits ovarian cancer cell proliferation, migration, and invasion; and induces apoptosis. Conclusion: The results suggest KIF2A could serve a valuable prognostic indicator in ovarian cancer and provide a rationale for treatment of ovarian cancer by targeting KIF2A via miR-206.
\end{abstract}

N. Sheng and Y.-Z. Xu contributed equally to this work.

\begin{tabular}{ll}
\hline Qing Ye & Department of Obstetrics and Gynecology, Affiliated Hospital of Nantong University \\
& Nantong, Jiangsu Province (China) \\
& Tel. +86 51381160622 , E-Mail ntyeging@163.com
\end{tabular}




\section{Introduction}

Ovarian cancer ranks fifth in cancer deaths among women, accounting for more deaths than any other cancer of the female reproductive system [1-3]. Studies have shown that cytoskeletal changes play an important role in the multi-factorial process of tumor cell metastasis [4] and that microtubule reduction and depolymerization are related to a tumor's metastatic potential [5].

In recent years, members of the kinesin-13 family have been identified, including kinesin family member 2A (KIF2A), KIF2B and mitotic centromere-associated kinesin (MCAK), which are all M-type nonmotile microtubule depolymerases [6-8]. It has been shown that these family members play an important role in regulating microtubule dynamics during mitotic progression $[7,9]$, especially intracellular transport, cell division and bipolar spindle assembly during spindle formation [7, 10, 11]. KIF2A, acting as a microtubule minus-end depolymerizing motor, was shown to be essential in assembling normal bipolar spindles [9]. Furthermore, it was demonstrated that KIF2A overexpression was associated with tumorigenesis and cancer progression and indicative of poor prognosis. For example, abnormal KIF2A expression was associated with metastasis and poor prognosis of breast cancer [12], glioma [13] and gastric cancer [14]. In our pervious study, we also found that KIF2A was also associated with ovarian cancer [15]. However, the mechanism of KIF2A and its prognostic role in ovarian cancer has not yet been explored.

MicroRNAs (miRNAs) are a class of small RNA molecules that regulate gene expression in a variety of ways, including translational repression, mRNA cleavage and deadenylation. Prior studies have shown that miR-206 is under expressed in many human cancers. In fact, some tumor genes can be inhibited in cancer cells by miR-206, such as SOX9 in nonsmall cell lung cancer (NSCLC) [16], MKL1/IL11 or Tbx3 in breast cancer [17, 18], CDK9 in hepatocarcinoma [19], EGFR and c-MET in head and neck squamous cell carcinoma [20], and ANXA2 in Osteosarcoma [21].

In this study, we demonstrated the expression of KIF2A in ovarian cancer tissues and investigated the miR-206 and its impact on KIF2A and ovarian cancer tumorigenesis and metastasis.

\section{Materials and Methods}

\section{Clinical specimens and immunohistochemical staining}

The study was approved by the Ethics Committee of Affiliated Hospital of Nantong University. A total of 108 cases of human ovarian cancer tissue samples were obtained from patients diagnosed with ovarian carcinoma that underwent initial surgery at Department of Obstetrics and Gynecology in Affiliated Hospital of Nantong University from 2005 to 2010 . All tissues were fixed in formalin and embedded in paraffin. None of the patients received any therapy against carcinoma prior to surgery. Patient clinical information was recorded in detail and the diagnoses were confirmed by at least 2 pathologists. Of the 108 ovarian cancer cases, 84 were serous carcinoma, 6 were mucinous carcinoma, 13 were endometrioid carcinoma and 5 were clear cell carcinoma. There were 60 cases in stage I/II and 48 cases in stage III/IV. In regards to histological grading, 78 cases were in high grade, and 30 cases were in low grade. 30 cases of fresh human ovarian cancer tissues and matched normal ovary tissues were collected for detection of KIF2A mRNA and protein via RT-qPCR and Western blot, respectively.

All paraffin-embedded tissues were cut into $4-\mu \mathrm{m}$ thickness sections. The Envision Plus/Horseradish Peroxidase system (DAKO, USA) was employed for immunohistochemical staining. The slides were incubated with rabbit anti-human KIF2A antibody (1:200 dilution) (Abcam, USA) overnight at $4{ }^{\circ} \mathrm{C}$, the negative control was the primary antibody replaced by PBS. After washing with phosphate-buffered saline (PBS), the slides were incubated using the Envision Plus secondary antibody for $30 \mathrm{~min}$, followed by diaminobenzidine for 5 min. Blinded pathologists independently evaluated and scored the immunohistochemistry results. Analysis was performed according to staining intensity and the relative abundance of positive cells. Staining intensity was graded as 0 (negative), 1 (weakly positive), 2 (moderately positive) and 3 (strongly positive). The percentage 


\section{Cellular Physiology Cell Physiol Biochem 2018;50:810-822 \begin{tabular}{l|l|l} 
and Biochemistry Published online: 23 October 2018 & $\begin{array}{l}\text { ○ 2018 The Author(s). Published by S. Karger AG, Basel } \\
\text { www.karger.com/cpb }\end{array}$ \\
\hline
\end{tabular}}

Sheng et al.: KIF2A is Suppressed by miR-206 in Ovarian Cancer

of positive cells was graded as $0(\leq 5 \%), 1$ (6-25\%), 2 (26-50\%), $3(51-75 \%)$ and $4(>75 \%)$. The scoring results were evaluated according to intensity and percentage score. KIF2A expression was defined as follows: "-" (negative, score of 0 ), "+" (weakly positive, score of 1-4), “++" (positive, score of 5-8), "+++" (strongly positive, score of 9-12).

\section{Cell culture and transfection}

The human ovarian cancer cells, A2780, SKOV3 and human embryonic kidney 293 (HEK293) cells were cultured in Dulbecco's modified Eagle's medium (DMEM) and supplemented with 10\% fetal bovine serum (FBS) (Thermo Fisher Scientific, USA) at $37^{\circ} \mathrm{C}$ in a cell culture incubator with $5 \% \mathrm{CO}_{2}$.

A human miR-206 mimic was used to simulate upregulation of endogenous miR-206. A scrambled sequence of miR-206 was used as a negative control (NC-miR). Lipofectamine $₫ 3000$ transfection reagent (Thermo Fisher Scientific, USA) was used for transfection of miRNA mimics or controls into cells according to the manufacturer's instructions. miR-206 mimics and negative controls were obtained from Biomics Biotechnologies Co., Ltd. (China).

\section{Dual-luciferase reporter (DLR) assay}

The DNA sequence complementary to the 3'UTR region of human KIF2A mRNA was obtained and constructed into the pGL3-vector (Promega, USA) as a miRNA reporting vector (WT-KIF2A-3'UTR) in order to evaluate miR-206 activity in cells. Mutant vectors were constructed using the KIF2A 3'UTR sequence with site mutations at either of the 2 miR-206-binding sites (Mut1-KIF2A-3'UTR and Mut2-KIF2A-3'UTR). Similarly, the negative control was constructed using the KIF2A 3'UTR sequence with site mutations at both of the two miR-206-binding sites (Mut3-KIF2A-3'UTR).

Briefly, HEK293 cells were cultured and plated on a 24-well plate and growth for $24 \mathrm{hrs,} \mathrm{then} \mathrm{WT-KIF2A-}$ 3'UTR or Mut-KIF2A-3'UTR was co-transfected with miR-206 mimics or NC-miR using Lipofectamine ${ }^{\circledR} 3000$ transfection reagent (Thermo Fisher Scientific, USA). Meanwhile, pRL-TK (Promega, USA) was co-transfected in each treatment as an internal control. $48 \mathrm{hrs}$ after transfection, HEK293 cells were collected and lysed. The luciferase activities of both firefly and Renilla in cell lysate were detected using the dual-luciferase reporter assay system (Promega, USA) according to the manufacturer's instructions.

\section{Real-time quantitative PCR (RT-qPCR)}

After treatment, total RNA of cells was extracted using TRIzol ${ }^{\circledR}$ reagent (Thermo Fisher Scientific, USA), and small RNA enriched with miRNAs was purified and enriched using the mirPremier ${ }^{\circledR}$ microRNA isolation kit (Sigma-Aldrich, USA) according to the manufacturers' instructions. Measurement of miR-206 expression in tissues or cells was performed by a stem-loop RT-qPCR method [22]. $\beta$-actin and U6 small RNA were used as an internal control for the detection of mRNA and miRNA, respectively. RT-qPCR reactions were carried out using the SYBR Green I One-Step RT-qPCR kit (Thermo Fisher Scientific, USA) according to the manufacturer's instructions. Relative mRNA and miRNA levels were analyzed by the $2^{-\Delta \Delta \mathrm{Ct}}$ method [23]. The primer sequences were as follows:

KIF2A forward: 5'-CCTGACCTTGTTCCTGATGAAG-3';

KIF2A reverse: 5'-TGCTGAACCAACCACTCTATTATC-3';

$\beta$-actin forward: 5'-CCACACCTTCTACAATGAG-3';

$\beta$-actin reverse: 5'-ATAGCACAGCCTGGATAG-3'.

\section{Western blot analysis}

After treatment for $48 \mathrm{hrs}$ as described above, in a 6-well plate, the cells were collected and lysed in cell RIPA lysis and extraction buffer (Thermo Fisher Scientific, USA) on ice. Total proteins were collected with centrifugation, separated by SDS-PAGE and transferred onto a PVDF membrane (Merckmillipore, USA). Then, the membrane was incubated with primary antibody, a rabbit anti-human KIF2A antibody (1:1, 000 dilution) (Abcam, USA) or a mouse anti-human $\beta$-actin antibody (1:5, 000 dilution) (Abcam, USA) as internal control. After washing with TBST 3 times, the membrane was incubated with secondary antibody, a horseradish peroxidase (HRP)-conjugated IgG (1:5, 000 dilution) (Abcam, USA) for 1.5 hrs at room temperature. After washing with TBST 3 times, the specific proteins were detected with Pierce ECL substrate (Thermo Fisher Scientific, USA). 


\section{Cellular Physiology Cell Physiol Biochem 2018;50:810-822 \begin{tabular}{ll|l} 
and Biochemistry Published onlIne: 23 October 2018 & $\begin{array}{l}\text { (c) } 2018 \text { The Author(s). Published by S. Karger AG, Basel } \\
\text { www.karger.com/cpb }\end{array}$ \\
\hline
\end{tabular}}

Sheng et al.: KIF2A is Suppressed by miR-206 in Ovarian Cancer

Cell proliferation assay

Cell proliferation was detected using the Cell Counting Kit-8 (CCK-8) (Sigma-Aldrich, USA). Briefly, before treatment, $1.5 \times 10^{4}$ cells/well were plated onto a 96-well plate and grown to $\sim 75 \%$ confluence for $24 \mathrm{hrs}$. With treatment at $0,24,48$ or $72 \mathrm{hrs}$, cells were washed 3 times with PBS (pH7.4) and incubated in $100 \mu \mathrm{l} /$ well of PBS and $10 \mu \mathrm{l}$ of the FCCK working solution at $37^{\circ} \mathrm{C}$ and $5 \% \mathrm{CO}_{2}$ for $30 \mathrm{~min}$. Finally, the fluorescence intensity of each well at $535 \mathrm{~nm}$ was measured using a fluorescence microplate reader (BioTek, USA).

Transwell assay

Cell migration and invasion were detected by a transwell assay. $2 \times 10^{5}$ cells/well were seeded onto a 24-well plate, grown for $24 \mathrm{hrs}$, treated for $48 \mathrm{hrs}$ and then suspended in DMEM at the density of $1 \times 10^{6}$ cells/ml. Transwell chambers (Corning, USA) were incubated with DMEM for $1 \mathrm{hr}$ before treatments. The upper and lower chambers were separated by an $8-\mu \mathrm{m}$ pore polycarbonate membrane. For cell invasion, an $8-\mu \mathrm{m}$ pore polycarbonate membrane should be coated with $50 \mu \mathrm{l}$ of $0.5 \mathrm{mg} / \mathrm{ml}$ matrigel (BD Biosciences, USA). $100 \mu \mathrm{l}$ cell suspension was added into each upper chamber with $600 \mu \mathrm{l}$ DMEM containing 10\% FBS or conditioned medium, which were the cell supernatant with different treatments for $48 \mathrm{hrs}$ as described above. 24 hrs after treatments, the cells on the top surface of the membrane were carefully removed using cotton swabs, and cells on the bottom surface were fixed in $10 \%$ formaldehyde for $30 \mathrm{~s}$ and stained with $0.5 \%$ crystal violet solution. After being washed with PBS, the cells on the top surface of the membrane were carefully removed again. Cells on the bottom surface of the membrane were counted in 3-5 random fields manually under microscope (Olympus, Japan).

\section{Cell apoptosis assay}

Annexin-V-FITC/Propidium Iodide (Sigma-Aldrich, USA) flow cytometry was utilized to evaluate cell apoptosis. Cells were plated on a 6-well plate, grown for $24 \mathrm{hrs}$ and then treated as described above for 48 hrs. $1 \times 10^{5}$ cells/well were harvested, washed 3 times with PBS, re-suspended in Annexin-binding buffer and then incubated with Annexin V-FITC conjugate and Propidium Iodide for $15 \mathrm{~min}$ at room temperature. Finally, the stained cells were analyzed by FCM (BD Biosciences, USA).

\section{Statistical analysis}

All experiments were performed independently 3 times. The data are shown as mean values \pm standard deviation (SD). Statistical analyses were performed using SPSS19.0 software. Significance between multiple groups was evaluated by one-way analysis of variance (ANOVA) followed by a Dunnett post hoc test. Comparisons between two groups were performed by Student's t-test. Spearman's correlation analysis was used to analyze the correlation between KIF2A and miR-206 expression. All $P$-values are based on a twotailed statistical analysis. $P<0.05$ was considered to indicate statistical significance.

\section{Results}

High KIF2A expression in patients with ovarian cancer

KIF2A mRNA expression was higher in ovarian cancer tissues than in normal tissues, as measured by RT-qPCR in tissues sourced from 30 cases of ovarian cancer and matched adjacent normal tissues $(P<0.01)$ (Fig. 1A). Similarly, KIF2A protein expression was higher in cancer tissues than in normal tissues, as measured by Western blot in ovarian cancer and paired adjacent normal tissues (Fig. 1B).

\section{Correlation of KIF2A expression and clinicopathological factors in ovarian cancer}

Immunohistochemical staining was performed to analyze KIF2A expression in ovarian cancer. The results showed that the moderate or strong staining of KIF2A in ovarian cancer tissues was $77.78 \%$ (84/108), while normal or weak staining was $22.22 \%(24 / 108)$ (Fig. 2 and Table 1). Thus, the expression of KIF2A was higher in cancer tissues than in normal tissues. The KIF2A expression was associated with clinicopathological factors in patients with ovarian cancer (Table 1). We found statistical differences between positive KIF2A 
A

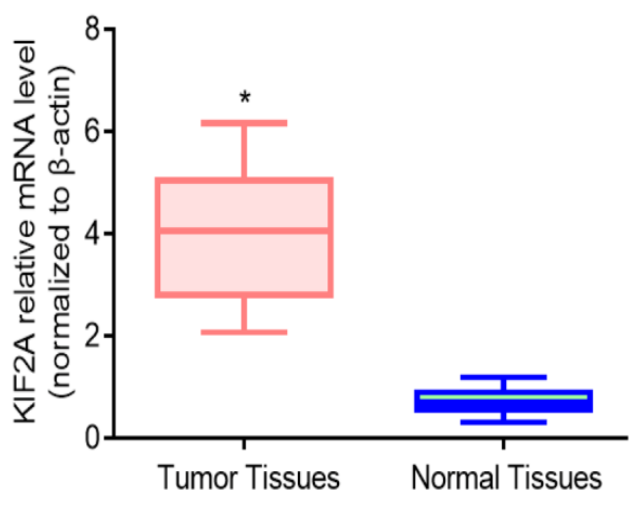

B
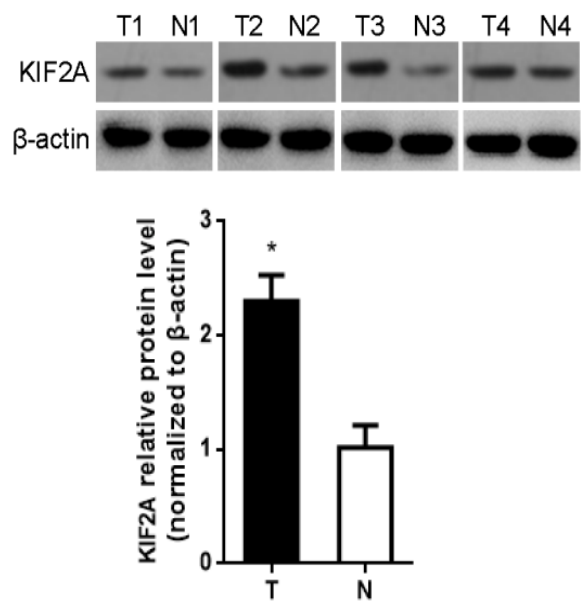

Fig. 1. KIF2A expression in ovarian cancer tissues and matched adjacent normal tissues. A. KIF2A expression was detected by RT-qPCR. B. KIF2A expression was detected by Western blot. ${ }^{*} \mathrm{P}<0.01$, compared with normal tissues.

Table 1. The association between KIF2A expression and clinical pathological factors in malignant ovarian cancer. *represents statistical significance, $\mathrm{P}<0.05$

\begin{tabular}{|c|c|c|c|c|c|}
\hline \multirow{2}{*}{ Clinicopathological parameters } & \multirow{2}{*}{ No. Case } & \multicolumn{2}{|c|}{ KIF2A Expression, $\mathrm{n}$} & \multirow{2}{*}{ Pearson $\mathrm{x}^{2}$} & \multirow{2}{*}{$\mathrm{P}$} \\
\hline & & $-/+$ & $++/+++$ & & \\
\hline Age & & & & 2.534 & 0.111 \\
\hline$\leq 50$ & 31 & 10 & 21 & & \\
\hline$>50$ & 77 & 14 & 63 & & \\
\hline Pathological type & & & & 4.942 & 0.176 \\
\hline Serous carcinoma & 84 & 15 & 69 & & \\
\hline Mucinous carcinoma & 6 & 3 & 3 & & \\
\hline Endometrioid carcinoma & 13 & 5 & 8 & & \\
\hline Clear cell carcinoma & 5 & 1 & 4 & & \\
\hline Surgical stage & & & & 4.725 & $0.030^{*}$ \\
\hline $\mathrm{I} / \mathrm{II}$ & 60 & 18 & 42 & & \\
\hline III/IV & 48 & 6 & 42 & & \\
\hline Grade & & & & 3.590 & 0.058 \\
\hline High grade carcinoma & 78 & 21 & 57 & & \\
\hline Low grade carcinoma & 30 & 3 & 27 & & \\
\hline Lymph node metastasis & & & & 1.606 & 0.205 \\
\hline No & 87 & 22 & 65 & & \\
\hline Yes & 21 & 2 & 19 & & \\
\hline Other organ metastasis & & & & 7.449 & $0.006^{*}$ \\
\hline No & 54 & 18 & 36 & & \\
\hline Yes & 53 & 6 & 47 & & \\
\hline Ascites & & & & 0.327 & 0.567 \\
\hline No & 62 & 15 & 47 & & \\
\hline Yes & 46 & 9 & 37 & & \\
\hline Five-year survival & & & & 5.510 & $0.019^{*}$ \\
\hline$<5$ years & 63 & 9 & 54 & & \\
\hline$\geq 5$ years & 45 & 15 & 30 & & \\
\hline
\end{tabular}


Fig. 2. KIF2A expression in ovarian cancer was analyzed by immunohistochemical staining. A. Weak KIF2A staining in the well differentiated serous carcinoma tissue. B. Strong KIF2A staining in the poorly differentiated serous carcinoma tissue.

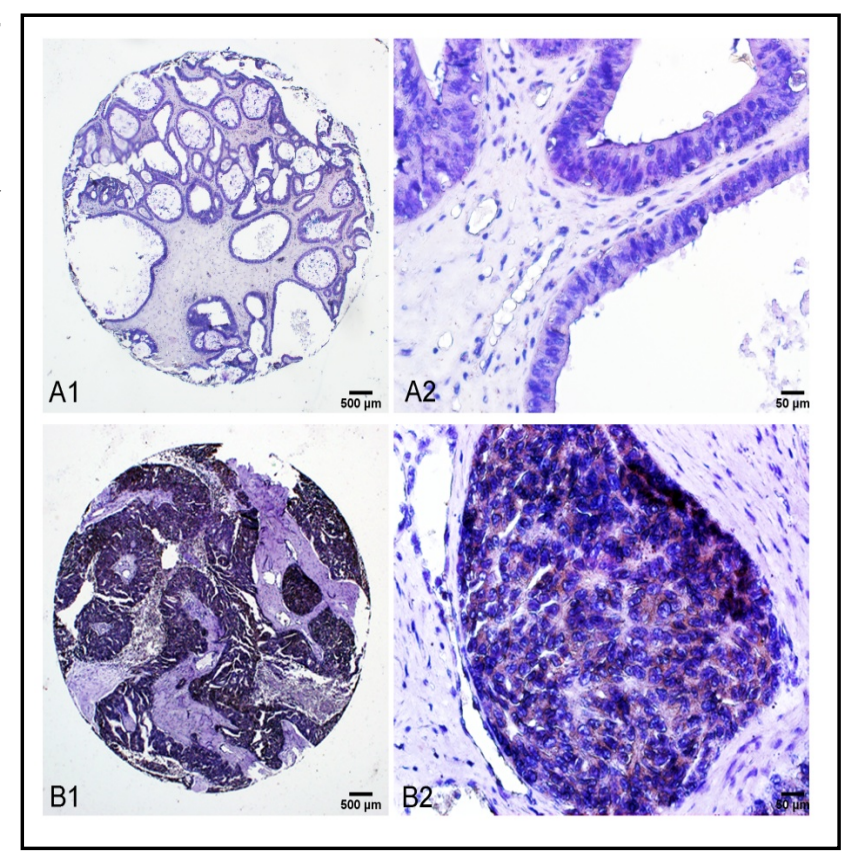

expression and the characteristics of surgical stage $(P=0.030)$, other organ metastasis $(P=0.006)$ and five-year survival $(P=0.019)$.

miR-206 expression profiles in ovarian cancer patients

miR-206 expression was detected by RT-qPCR in the tissues of 30 ovarian cancer cases and matched adjacent normal tissues. miR-206 was significantly decreased in ovarian cancer tissues versus normal tissues $(P<0.01)$ (Fig. 3). Spearman's correlation analysis showed that miR-206 correlated negatively with KIF2A expression $(r=-0.704, P<0.01)$ (Fig. 4).

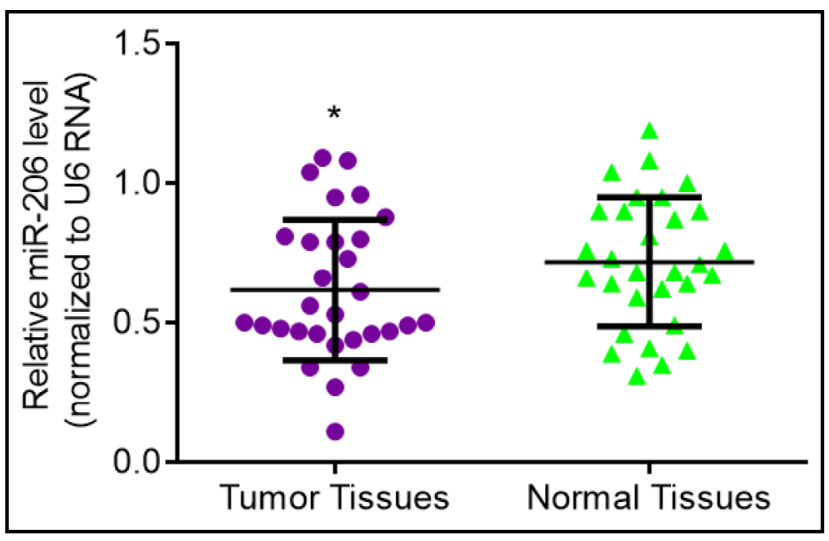

Fig. 3. miR-206 expression in ovarian cancer patients was detected by RT-qPCR in 30 cases of ovarian cancer tissues and matched adjacent normal tissues. ${ }^{*} \mathrm{P}<0.01$, compared with normal tissues.

\section{miR-206 targeted the 3'UTR of KIF2A mRNA}

As predicted by TargetScan online software (http://www.targetscan.org), KIF2A had 2 miR-206 binding sites, at positions 514 and 658 (Fig. 5A). The dual-luciferase reporter (DLR) vectors of WT-KIF2A-3'UTR and Mut-KIF2A-3'UTR were constructed in order to verify the inhibitory effect of miR-206 (Fig. 5B). When HEK293 cells were co-transfected with miR206 mimics and WT-KIF2A-3'UTR, an obvious decrease in luciferase activity was observed compared to cells co-transfected with NC-miR and WT-KIF2A-3'UTR, Mut1-KIF2A-3'UTR, Mut2-KIF2A-3'UTR or Mut3-KIF2A-3'UTR $(P<0.05$, Fig. 5C). Luciferase activities were also decreased in cells that were co-transfected with miR-206 mimics and Mut1-KIF2A-3'UTR or Mut2-KIF2A-3'UTR. However, the inhibitory effects were not significant as compared to cells co-transfected with miR-206 and WT-KIF2A-3'UTR. 


\section{Cellular Physiology Cell Physiol Biochem 2018;50:810-822}

\begin{tabular}{l|l|l} 
DOl: 10.1159/000494467 & $\begin{array}{l}\text { O 2018 The Author(s). Published by S. Karger AG, Basel } \\
\text { www.karger.com/cpb }\end{array}$
\end{tabular}

Sheng et al.: KIF2A is Suppressed by miR-206 in Ovarian Cancer

Fig. 4. The correlation between KIF2A and miR206 expression in ovarian cancer tissues.
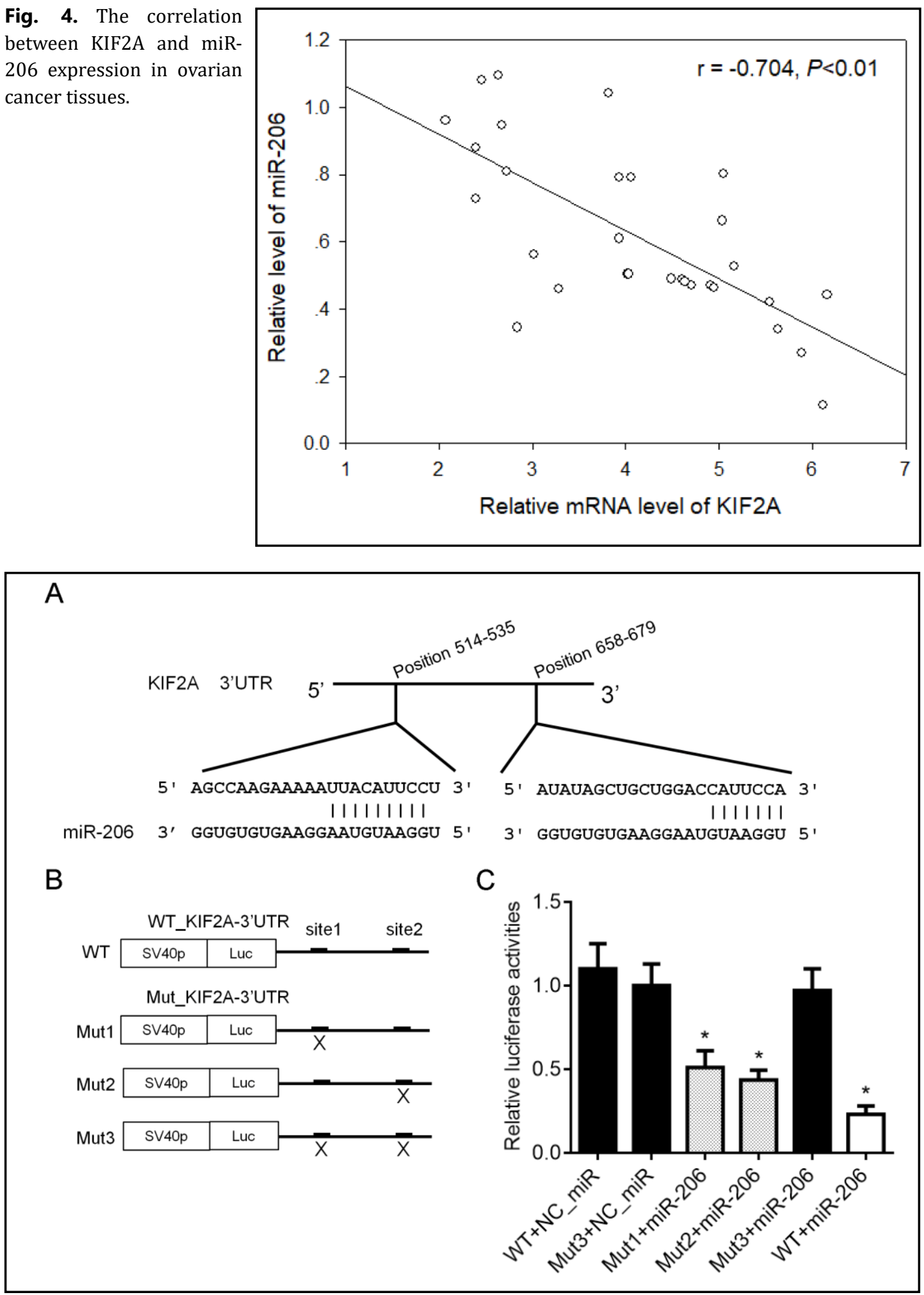

Fig. 5. miR-206 targeted the 3'UTR of KIF2A mRNA. A. As predicted by software, there were 2 binding sites for miR-206 at positions 514 and 658 of KIF2A. B. The constructed dual-luciferase reporter vectors of WT-KIF2A-3'UTR, Mut1-KIF2A-3'UTR Mut2-KIF2A-3'UTR and Mut3-KIF2A-3'UTR. C. miR-206 inhibition of KIF2A was detected by DLR assay, ${ }^{*} \mathrm{P}<0.05$, NC-miR transfected with WT-KIF2A-3'UTR or Mut3-KIF2A3'UTR vs. miR-206 transfected with Mut1-KIF2A-3'UTR, Mut2-KIF2A-3'UTR or WT-KIF2A-3'UTR. 


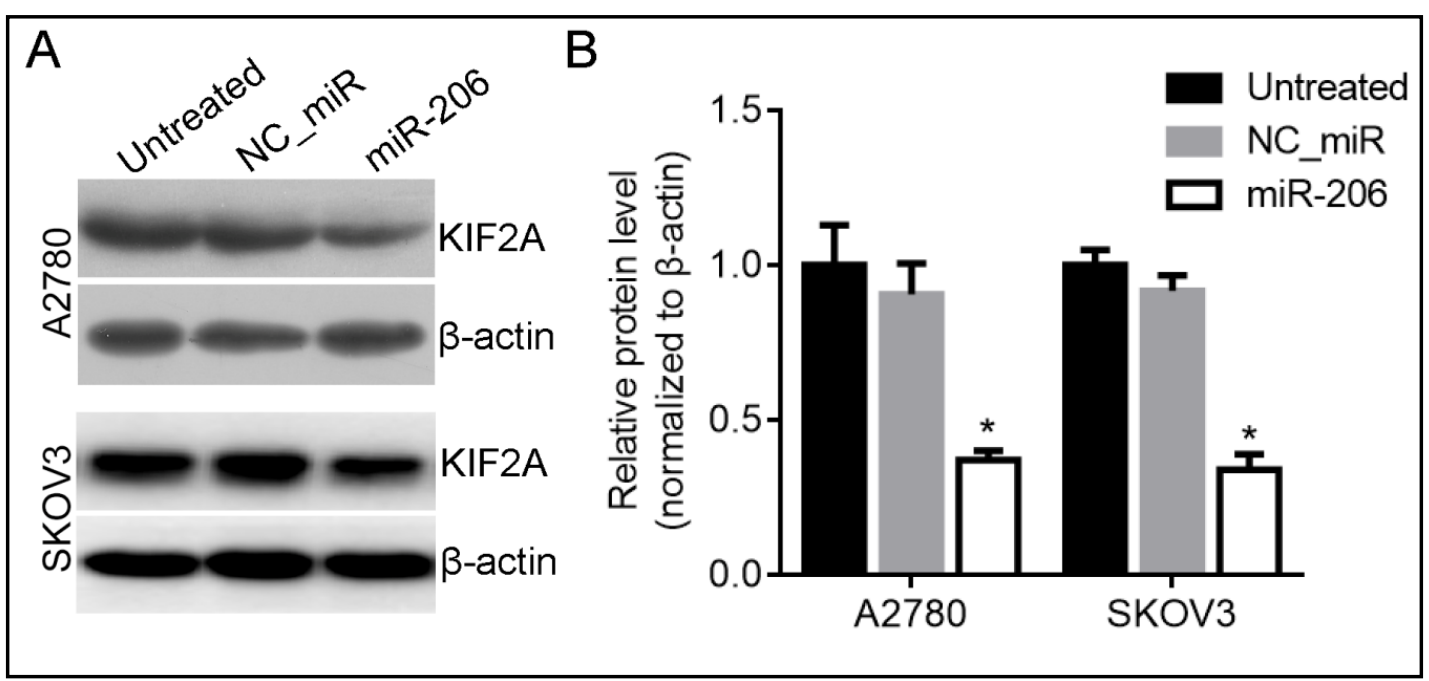

Fig. 6. KIF2A expression was inhibited by miR-206 in ovarian cancer cells. A. KIF2A protein was decreased after A2780 and SKOV3 cells were treated with miR-206, as detected by Western blot. B. Relative KIF2A protein inhibited by miR-206 in A2780 and SKOV3 cells. ${ }^{*} \mathrm{P}<0.05$, miR-206- vs. NC-miR-treated cells.

\section{miR-206 inhibited KIF2A in ovarian cancer cells}

miR-206 significantly inhibited KIF2A expression in A2780 and SKOV3 cells, as measured by Western blot and compared to NC-miR-treated cells $(P<0.05)$ (Fig. 6).

Effect of miR-206-mediatedKIF2A inhibition on ovarian cancer cell growth

A Cell Counting Kit-8 (CCK8) assay was utilized in order to investigate the inhibitory effects of miR-206 on ovarian cancer cell growth. miR206 clearly inhibited the growth of A2780 and SKOV3 cells at 48 and 72 hrs versus cells treated with NC-miR $(P<0.05)$ (Fig. 7).

Effect of miR-206-mediated KIF2A inhibition on ovarian cancer cell invasion and migration

A matrigel ${ }^{\mathrm{TM}}$ invasion assay and a transwell assay were utilized to evaluate the effect of miR-206mediated KIF2A inhibition on ovarian cancer cell invasion and migration, respectively. miR-206 treatment significantly decreased A2780 and SKOV3 cell migration and invasion versus cells treated with NC-miR $(P<0.05)$ (Fig. 8).

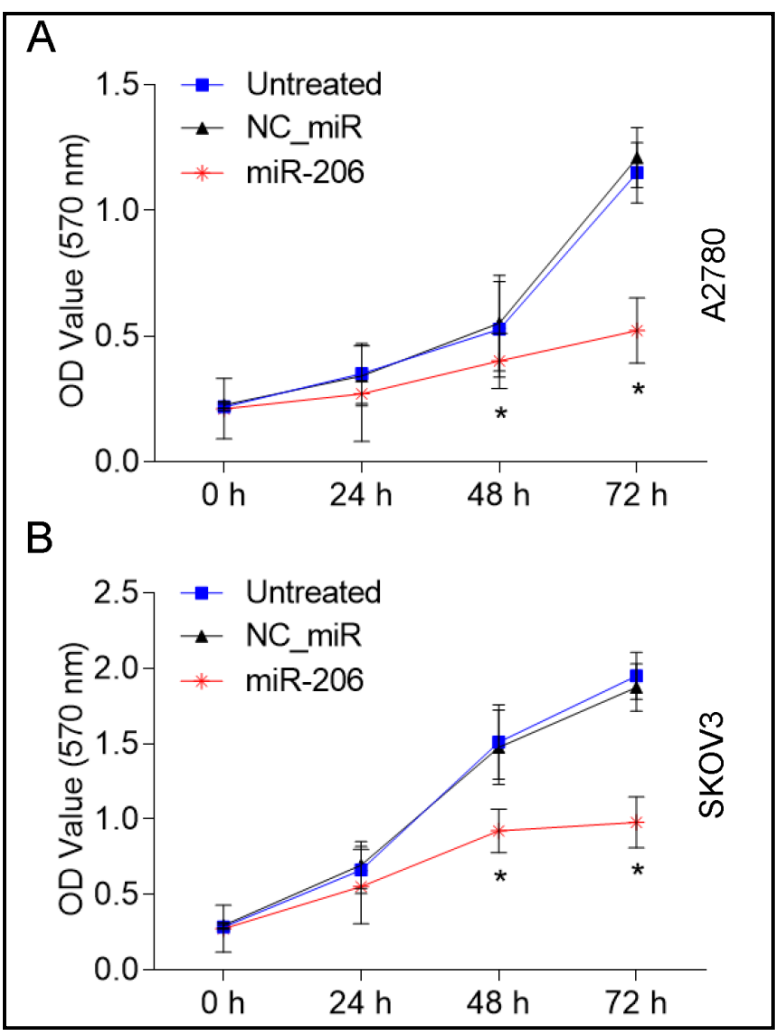

Fig. 7. Effect of miR-206 treatment on ovarian cancer cell growth. A. The growth of A2780 cell affected by miR-206. B. The growth of SKOV3 cell affected by miR-206. ${ }^{*} \mathrm{P}<0.05$, miR-206- vs. NC-miR-treated cells. 


\section{Cellular Physiology Cell Physiol Biochem 2018;50:810-822

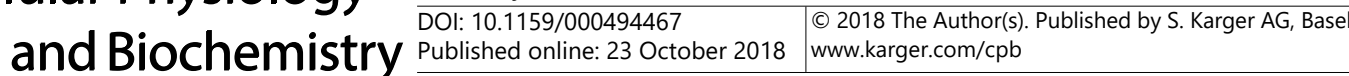

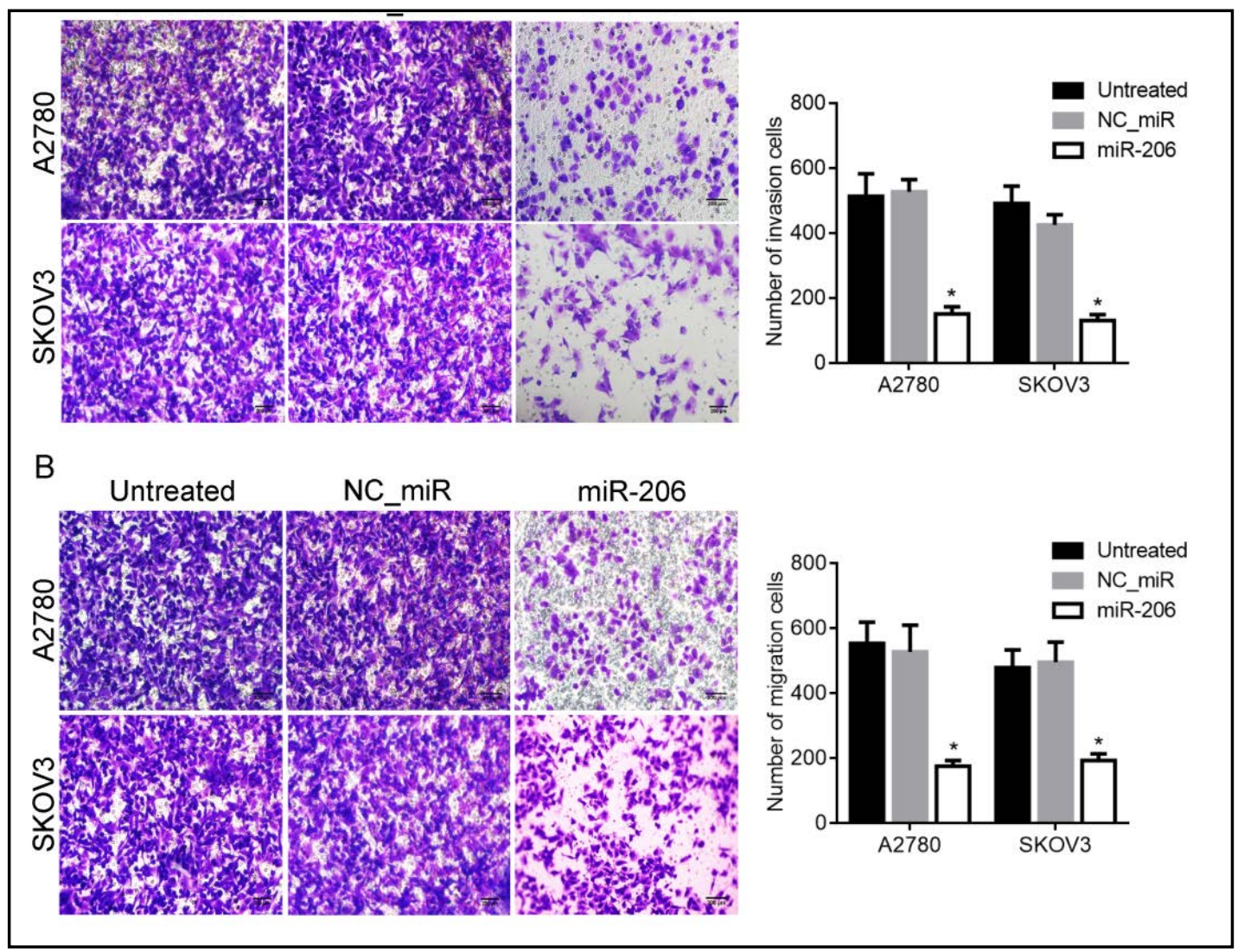

Fig. 8. Effect of miR-206 treatment on ovarian cancer cell invasion and migration. A. miR-206 inhibited the invasion abilities of A2780 and SKOV3 cells. B. miR-206 inhibited the migration abilities of A2780 and SKOV3 cells. ${ }^{*} \mathrm{P}<0.05$, miR-206- vs. NC-miR-treated cells.

Effect of miR-206-mediated KIF2A inhibition on ovarian cancer cell apoptosis

Annexin-V-FITC/Propidium Iodide flow cytometry was utilized to evaluate the effect of miR-206-mediated KIF2A inhibition on ovarian cancer cell apoptosis. Treatment with miR206 for 48 hrs significantly increased apoptosis both of A2780 and SKOV3 cells versus cells treated with NC-miR $(P<0.05)$ (Fig. 9). 


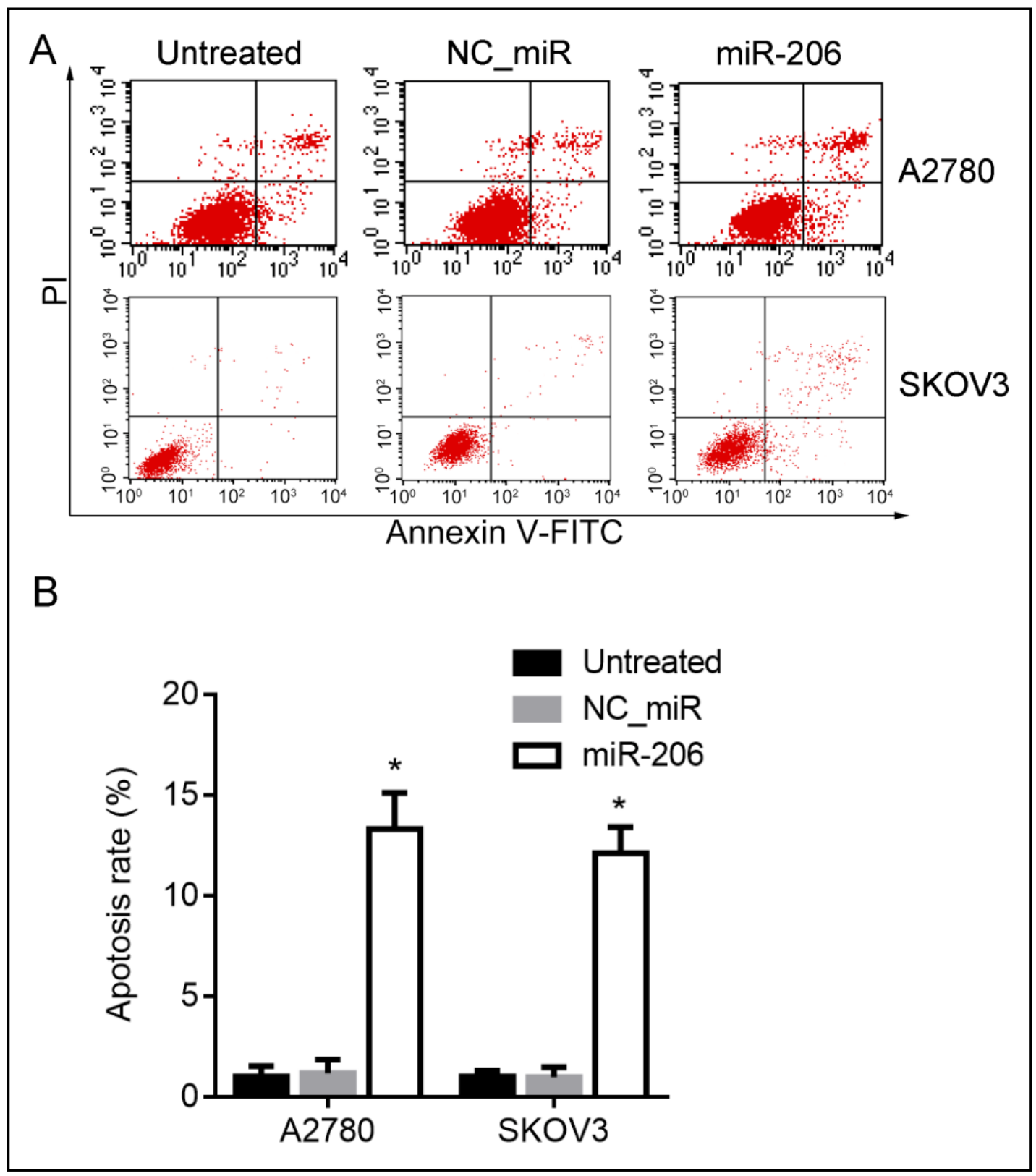

Fig. 9. Effect of miR-206 treatment on ovarian cancer cell apoptosis. A. Apoptosis of miR-206-treated A2780 and SKOV3 cells as detected by FCM after Annexin V-FITC/Propidium Iodide double staining. B. The apoptosis rate of A2780 and SKOV3 cells after miR-206 treatment. ${ }^{*} \mathrm{P}<0.05$, compared with NC-miR treated cells.

\section{Discussion}

KIF2A, a type of motor protein with an oncogenic role, is overexpressed in many human malignancies [12-15]. KIF2A is associated with the development and progression of various human cancers and can be an indicator of poor prognosis. In recently studies showed that, KIF2A overexpression was significantly correlated to clinicopathologic characteristics and poor prognoses in patients with gastric cancer [14], diffuse large B cell lymphoma [24], laryngeal squamous cell carcinoma [25]. In hepatocellular carcinoma, KIF2A expression was positively associated with biomarkers for cell invasion and migration, and its overexpression 
was associated with overall survival time [26]. In our study, we demonstrated that KIF2A expression was increased in human ovarian cancer patients and in tumor tissues. We also found that high KIF2A expression and low KIF2A expression groups presented significant differences in surgical stage, organ metastasis and five-year survival.

Increased expression of miR-206 increased cancer cell apoptosis and inhibited proliferation, migration and invasion. Prior to this study, the relationship between miR-206 and KIF2A had not been reported. Utilizing TargetScan online software, KIF2A was predicted to have two miR-206 binding sites in the 3'UTR of the KIF2A mRNA. We also demonstrated that miR-206 was under expressed in ovarian cancer tissues and that miR-206 expression correlated negatively with KIF2A expression $(r=-0.704, P<0.01)$. miR-206 inhibition of KIF2A was further confirmed by a dual-luciferase reporter assay. We utilized a miR-206 mimic to simulate an increase in miR-206 expression in an ovarian cancer cell lines, which was clearly effective in decreasing KIF2A protein levels.

The previous studies have been reported that the proliferation and migration of cancers cells were promoted and apoptosis were inhibited by high expression of KIF2A which predicted poor prognosis in several cancers, such as in breast cancer [12], glioma [13], squamous cell carcinoma of the oral tongue [27] and head and neck squamous cell carcinoma [28]. Additionally, recent studies have also shown that miR-206, a tumor suppressor miRNA that targets several oncogenes, was under expressed in many tumors [16-20], miR-206 inhibited cell proliferation, invasion and/or apoptosis by targeting SOX9 in lung cancer [16], Tbx3 in breast cancer [18], CDK9 in hepatocellular carcinoma [19], HDAC6in head and neck squamous cell carcinoma [20], ANXA2 in osteosarcoma [21], Bcl-2 in colorectal cancer [29], CXCL11 in prostate cancer [30], HDAC6 in squamous cell carcinoma [27], and cell metastasis by targeting MKL1/IL11 in breast cancer [17], FMNL2 in colorectal cancer [31], CXCL11 in prostate cancer [30]. And serum miR-206 might serve as a diagnostic biomarker in cancers, such as gastric cancer [32], renal cell carcinoma [33], also as a novel prognostic and therapeutics in cervical cancer [34]. In the study, we showed that inhibition of KIF2A by miR-206 decreased ovarian cancer cells A2780 and SKOV3 proliferation, migration and invasion and increased apoptosis.

\section{Conclusion}

We confirmed that miR-206 could inhibit the biological behavior of ovarian cancer by inhibition of KIF2A mRNA with two target sites. Taken together, our findings demonstrate KIF2A as a potential prognostic indicator in ovarian cancer and a rationale for treatment of ovarian cancer by targeting KIF2A via miR-206.

\section{Acknowledgements}

This work was supported by grants from the Science Foundation of Nantong City, Jiangsu Province, China (No. MS22015106).

\section{Disclosure Statement}

All authors declare that they have no conflicts of interest.

\section{References}

1 Siegel RL, Miller KD, Jemal A: Cancer statistics, 2016. CA Cancer J Clin 2016;66:7-30. 


\section{Cellular Physiology Cell Physiol Biochem 2018;50:810-822 \begin{tabular}{l|l|l|l} 
DOI: 10.1159/000494467 & O 2018 The Author(s). Published by S. Karger AG, Basel \\
www.karger.com/cpb
\end{tabular}

-2 Wright JD, Chen L, Tergas AI, Patankar S, Burke WM, Hou JY, Neugut AI, Ananth CV, Hershman DL: Trends in relative survival for ovarian cancer from 1975 to 2011. Obstet Gynecol 2015;125:1345-1352.

- 3 Rojas V, Hirshfield KM, Ganesan S, Rodriguez-Rodriguez L: Molecular Characterization of Epithelial Ovarian Cancer: Implications for Diagnosis and Treatment. Int J Mol Sci 2016;17:2113.

4 Olson MF, Sahai E: The actin cytoskeleton in cancer cell motility. Clin Exp Metastasis 2009;26:273-287.

5 Chandrasekaran G, Tátrai P, Gergely F: Hitting the brakes: targeting microtubule motors in cancer. $\mathrm{Br} \mathrm{J}$ Cancer 2015;113:693-698.

6 Moore A, Wordeman L: The mechanism, function and regulation of depolymerizing kinesins during mitosis. Trends Cell Biol 2004;14:537-546.

7 Manning AL, Ganem NJ, Bakhoum SF, Wagenbach M, Wordeman L, Compton DA: The kinesin-13 proteins KIF2A, KIF2B, and KIF2C/MCAK have distinct roles during mitosis in human cells. Mol Biol Cell 2007;18:2970-2979.

-8 Ishikawa K, Kamohara Y, Tanaka F, Haraguchi N, Mimori K, Inoue H, Mori M: Mitotic centromere-associated kinesin is a novel marker for prognosis and lymph node metastasis in colorectal cancer. Br J Cancer 2008;98:1824-1829.

9 Ganem NJ, Compton DA: The KinI kinesin Kif2a is required for bipolar spindle assembly through a functional relationship with MCAK. J Cell Biol 2004;166:473-478.

10 Wittmann T, Hyman A, Desai A: The spindle: a dynamic assembly of microtubules and motors. Nat Cell Biol 2001;3:E28-34.

11 Shimo A, Tanikawa C, Nishidate T, Lin ML, Matsuda K, Park JH, Ueki T, Hirata K, Fukuda M, Nakamura $\mathrm{Y}$, Katagiri T: Involvement of kinesin family member $2 \mathrm{C} /$ mitotic centromere-associated kinesin overexpression in mammary carcinogenesis. Cancer Sci 2008;99:62-70.

12 Wang J, Ma S, Ma R, Qu X, Liu W, Lv C, Zhao S, Gong Y: KIF2A silencing inhibits the proliferation and migration of breast cancer cells and correlates with unfavorable prognosis in breast cancer. BMC Cancer 2014;14:461.

13 Zhang X, Ma C, Wang Q Liu J, Tian M, Yuan Y, Li X, Qu X: Role of KIF2A in the progression and metastasis of human glioma. Mol Med Rep 2016;13:1781-1787.

14 Zhang S, Huang F, Wang Y, Song Q, Yang X, Wu H: KIF2A Overexpression and Its Association with Clinicopathologic Characteristics and Poor Prognoses in Patients with Gastric Cancer. Dis Markers 2016;2016:7484516.

15 Wang D, Zhu H, Ye Q, Wang C, Xu Y: Prognostic Value of KIF2A and HER2-Neu Overexpression in Patients With Epithelial Ovarian Cancer. Medicine (Baltimore) 2016;95:e2803.

16 Zhang YJ, Xu F, Zhang YJ, Li HB, Han JC, Li L: miR-206 inhibits non small cell lung cancer cell proliferation and invasion by targeting SOX9. Int J Clin Exp Med 2015;8:9107-9113.

17 Samaeekia R, Adorno-Cruz V, Bockhorn J, Chang YF, Huang S, Prat A, Ha N, Kibria G, Huo D, Zheng H, Dalton R, Wang Y, Moskalenko GY, Liu H: miR-206 Inhibits Stemness and Metastasis of Breast Cancer by Targeting MKL1/IL11 Pathway. Clin Cancer Res 2017;23:1091-1103.

-18 Amir S, Simion C, Umeh-Garcia M, Krig S, Moss T, Carraway KL 3rd, Sweeney C: Regulation of the T-box transcription factor Tbx3 by the tumour suppressor microRNA-206 in breast cancer. Br J Cancer 2016;114:1125-1134.

-19 Pang C, Huang G, Luo K, Dong Y, He F, Du G, Xiao M, Cai W: miR-206 inhibits the growth of hepatocellular carcinoma cells via targeting CDK9. Cancer Med 2017;6:2398-2409.

20 Koshizuka K, Hanazawa T, Fukumoto I, Kikkawa N, Matsushita R, Mataki H, Mizuno K, Okamoto Y, Seki N: Dual-receptor (EGFR and c-MET) inhibition by tumor-suppressive miR-1 and miR-206 in head and neck squamous cell carcinoma. J Hum Genet 2017;62:113-121.

21 Pan BL, Tong ZW, Wu L, Pan L, Li JE, Huang YG, Li SD, Du SX, Li XD: Effects of MicroRNA-206 on Osteosarcoma Cell Proliferation, Apoptosis, Migration and Invasion by Targeting ANXA2 Through the AKT Signaling Pathway. Cell Physiol Biochem 2018;45:1410-1422.

-22 Chen C, Ridzon DA, Broomer AJ, Zhou Z, Lee DH, Nguyen JT, Barbisin M, Xu NL, Mahuvakar VR, Andersen MR, Lao KQ, Livak KJ, Guegler KJ: Real-time quantification of microRNAs by stem-loop RT-PCR. Nucleic Acids Res 2005;33:e179.

-23 Livak KJ, Schmittgen TD: Analysis of relative gene expression data using real-time quantitative PCR and the 2(-Delta Delta C(T)) Method. Methods 2001;25:402-408. 


\section{Cellular Physiology Cell Physiol Biochem 2018;50:810-822

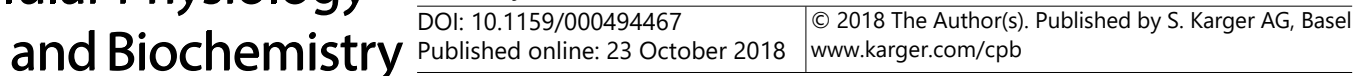

Sheng et al.: KIF2A is Suppressed by miR-206 in Ovarian Cancer

24 Zhang Y, You X, Liu H, Xu M, Dang Q, Yang L, Huang J, Shi W: High KIF2A expression predicts unfavorable prognosis in diffuse large B cell lymphoma. Ann Hematol 2017;96:1485-1491.

-25 Zhang Q, Zhang W, Zhang J, Xu H, You Y: Aberrant Kif2a and Ki67 expression predicts poor survival in laryngeal squamous cell carcinoma. Auris Nasus Larynx 2016;43:433-439.

-26 Chen J, Li S, Zhou S, Cao S, Lou Y, Shen H, Yin J, Li G: Kinesin superfamily protein expression and its association with progression and prognosis in hepatocellular carcinoma. J Cancer Res Ther 2017;13:651659.

27 Wang K, Lin C, Wang C, Shao Q Gao W, Song B, Wang L, Song X, Qu X, Wei F: Silencing Kif2a induces apoptosis in squamous cell carcinoma of the oral tongue through inhibition of the PI3K/Akt signaling pathway. Mol Med Rep 2014;9:273-278.

28 Liu F, Zhao X, Qian Y, Zhang J, Zhang Y, Yin R: MiR-206 inhibits Head and neck squamous cell carcinoma cell progression by targeting HDAC6 via PTEN/AKT/mTOR pathway. Biomed Pharmacother 2017;96:229-237.

29 Meng X, Fu R: miR-206 regulates 5-FU resistance by targeting Bcl-2 in colon cancer cells. Onco Targets Ther 2018;11:1757-1765.

-30 Wang Y, Xu H, Si L, Li Q Zhu X, Yu T, Gang X: MiR-206 inhibits proliferation and migration of prostate cancer cells by targeting CXCL11. Prostate 2018;78:479-490.

-31 Ren XL, He GY, Li XM, Men H, Yi LZ, Lu GF, Xin SN, Wu PX, Li YL, Liao WT, Ding YQ Liang L: MicroRNA-206 functions as a tumor suppressor in colorectal cancer by targeting FMNL2. J Cancer Res Clin Oncol 2016;142:581-592.

32 Hou CG, Luo XY, Li G: Diagnostic and Prognostic Value of Serum MicroRNA-206 in Patients with Gastric Cancer. Cell Physiol Biochem 2016;39:1512-1520.

-33 Heinemann FG, Tolkach Y, Deng M, Schmidt D, Perner S, Kristiansen G, Müller SC, Ellinger J: Serum miR122-5p and miR-206 expression: non-invasive prognostic biomarkers for renal cell carcinoma. Clin Epigenetics 2018;10:11.

-34 Chen AH, Qin YE, Tang WF, Tao J, Song HM, Zuo M: MiR-34a and miR-206 act as novel prognostic and therapy biomarkers in cervical cancer. Cancer Cell Int 2017;17:63. 